\title{
Coping with Polypharmacology by Computational Medicinal Chemistry
}

\author{
Gisbert Schneider*, Daniel Reker, Tiago Rodrigues, and Petra Schneider
}

\begin{abstract}
Predicting the macromolecular targets of drug-like molecules has become everyday practice in medicinal chemistry. We present an overview of our recent research activities in the area of polypharmacology-guided drug design. A focus is put on the self-organizing map (SOM) as a tool for compound clustering and visualization. We show how the SOM can be efficiently used for target-panel prediction, drug re-purposing, and the design of focused compound libraries. We also present the concept of virtual organic synthesis in combination with quantitative estimates of ligand-receptor binding, which we used for de novo designing target-selective ligands. We expect these and related approaches to enable the future discovery of personalized medicines.
\end{abstract}

Keywords: De novo design · Drug discovery · Machine learning · Molecular informatics · Self-organizing map

\section{Introduction}

For a long time, the ultimate goal of computer-assisted drug design has been to exclusively generate target-selective new chemical entities (NCEs), but it has been realized that synthetic drugs and bioactive natural products often interact with multiple macromolecular targets, which can be the reason for side-effect liabilities but also represent an integral aspect of their pharmacological mode of action. ${ }^{[1,2]}$ In this context it is common to distinguish between a compound's on-and off-targets. ${ }^{[3]}$ While target selectivity certainly remains a necessary and beneficial property of many drugs, there are therapeutic opportunities for pharmacologically active compounds with multi-target engagement. ${ }^{[4]}$ Such examples are central-nervous systems agents, anti-inflammatory drugs, anti-tumor agents, and novel antibiotics. ${ }^{[5]}$ Multi-target drugs also bear potential for treating currently incurable and neglected diseases. ${ }^{[6]}$ Numerous drug re-purposing ('re-positioning') opportunities arise from addressing the off-target activities of marketed medicines. ${ }^{[7]}$ In fact, a recent survey states that drug re-positioning accounts for approximately $30 \%$ of the newly U.S. Food and Drug Administration (FDA)-approved drugs and vaccines. ${ }^{[8]}$

Computational approaches not only help with the assessment of potential

\footnotetext{
${ }^{\star}$ Correspondence: Prof. Prof. adj. Dr. G. Schneider Eidgenössische Technische Hochschule

Department of Chemistry and Applied Biosciences

Computer-Assisted Drug Design

Vladimir-Prelog-Weg 4

$\mathrm{CH}-8093$ Zürich, Switzerland

Tel.: +41446337438

E-mail: gisbert.schneider@pharma.ethz.ch
}

polypharmacological activities but also in the early recognition of undesired sideeffects of small molecules. ${ }^{[9]}$ The idea is to predict the macromolecular targets for a given molecule from its chemical structure. There are several commercial tools for target prediction but also a number of publicly accessible methods (Table 1). With only few exceptions, these methods are exclusively ligand-based, which means they do not rely on three-dimensional (3D) receptor-ligand complementarity and may be used in the absence of receptor structure information. In fact, ligand-based methods often outperform 3D approaches with regard to prediction accuracy and target coverage, which is largely owed to imperfect
3D scoring functions for $\Delta G$ estimation. ${ }^{[17]}$ In particular large-scale applications require fast computing for millions of compounds, rendering the currently available advanced free energy estimators impracticable for polypharmacology prediction. The Chemical Similarity Principle states that structurally similar compounds tend to possess similar properties and biological activity, ${ }^{[18]}$ implying similar target engagement by structurally similar compounds. However, the appropriate definition of chemical similarity and choice of similarity metric are context-dependent. The most popular molecular descriptors are substructure similarity indices based on molecular graph representations as well as shape and

Table 1. Selection of publically accessible software tools for target prediction.

\section{Name}

Similarity Ensemble Approach(SEA)

SOM-based

Prediction of Drug

Equivalence Relationships (SPiDER)

Semantic Link

Association

Prediction (SLAP)

Prediction of

Activity Spectra for

Substances (PASS)

TargetHunter

SuperPred

TarFisDock

WWW link (URL)
http://sea.bkslab.org/
http://modlab-cadd.ethz.
ch/software/spider/

http://cheminfov. informatics.indiana. edu:8080/slap/ www.pharmaexpert.ru/ passonline/

http://www.cbligand. org/TargetHunter

http://prediction.charite. de/

http://www.dddc.ac.cn/ tarfisdock/

\section{Description}

Reference

Set-wise chemical similarity among protein ligands

Set-wise pharmacophore and physicochemical similarity among druglike bioactive compounds

Multi-model similarity assessment

Substructure-based similarity

Substructure-based similarity

Multi-step substructure and 3D similarity among known ligands

3D ligand docking into protein targets 
pharmacophore descriptors, all of which have been successfully employed for target prediction. ${ }^{[19]}$ An overview of these methods for de novo drug design can be found elsewhere. ${ }^{[1]}$ Here we present some of our own methodological concepts and recent developments in this research area.

\section{Target Panel Prediction and Ligand 'Deorphaning'}

The self-organizing map (SOM) concept provides a robust classifier for target panel assessment. ${ }^{[20]}$ Originally conceived of by Kohonen in $1984^{[21]}$ and pioneered in chemistry by Gasteiger and coworkers in the 1990s, ${ }^{[22]}$ we have established SOMs for clustering and visualizing chemical space. When used with a molecular pharmacophore representation (CATS, Chemically Advanced Template Search), ${ }^{[23]}$ these have proven useful for identifying common traits in molecules that make them likely to engage in similar interactions with biomacromolecules. ${ }^{[24]}$ This pattern recognition capacity originates from the ability of SOMs to identify regions (clusters) of chemical space that are characterized by a common but distinct pharmacophore pattern ('activity islands', Fig. 1). ${ }^{[25]}$ The beauty of this unsupervised tesselation approach lies in its simplicity and low data requirements. In contrast to many other methodologies that aim at predicting the polypharmacology of chemical entities, the SOM neither requires a three-dimensional model of the macromolecular binding pocket nor large sets of known ligand structures.

The idea of SOM-based target prediction is straightforward: Take a set of known drugs (so-called reference compounds) with known target annotations and group them according to common features (Fig. 2A). Each resulting cluster contains similar reference compounds, and potential targets of a co-clustered query compound are inferred from the reference target annotations. However, the clustering does not allow prioritization of target hypotheses for a specific cluster per se. To overcome this limitation, we have established a protocol that relies on the statistical interpretation of molecular similarities. ${ }^{[27]} \mathrm{A}$ molecular similarity score is transformed into a $p$ value using a background distribution of similarities between reference compounds that are not annotated to have a common target ('inter-target' distribution, Fig. 2B). Thereby, we obtain an estimate of the false-positive prediction probability. By averaging the $p$ values associated with the similarity between a query compound and the co-clustered references with the same target annotation, every target prediction obtains a confidence score.

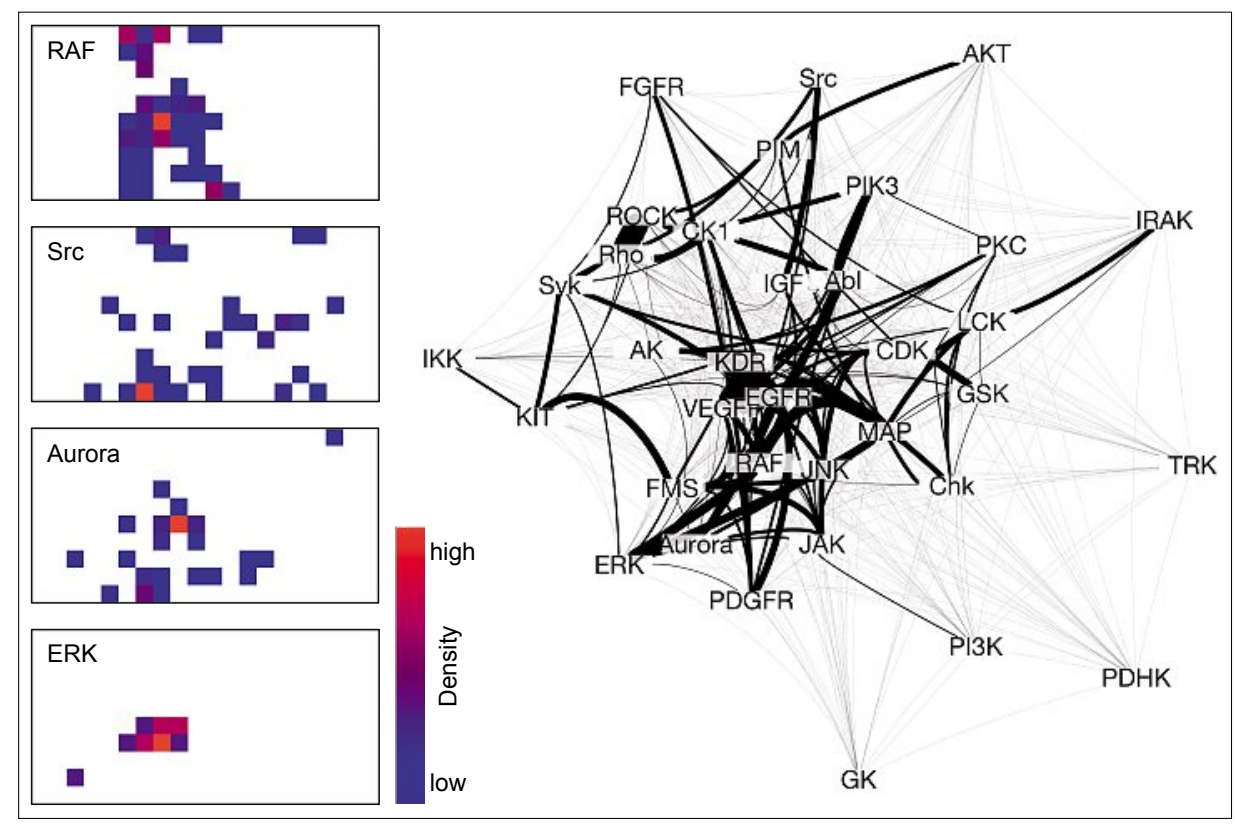

Fig. 1. The left panel shows four projections of different sets of known kinase inhibitors (RAF, Src, Aurora, ERK) in the same SOM. The SOM was generated using a total of 15,041 drugs and drug-like bioactive molecules with known targets. ${ }^{[26]}$ Compounds were represented in terms of their topological pharmacophoric features (CATS2 descriptor). ${ }^{[2,27]}$ For example, the ERK inhibitors form a well confined activity island on the map. By comparing the distribution of the inhibitor sets on the SOM, the similarity between the sets may be assessed, as shown by the SOM-based network graph of kinase targets (right panel). Strong connections indicate a strong correlation between the respective SOM projections. In this way, potential multi-target engagement by a ligand can be estimated.
We have previously applied the SOM in several prospective applications, e.g. combinatorial library profiling and focused library design. ${ }^{[28]}$ Recently we have prioritized a combinatorial library for a polypharmacological anti-cancer application. Certain Ugi-type three-component products were successfully associated with inhibition of both DNA topoisomerase and phosphoinositide 3-kinase, a com-

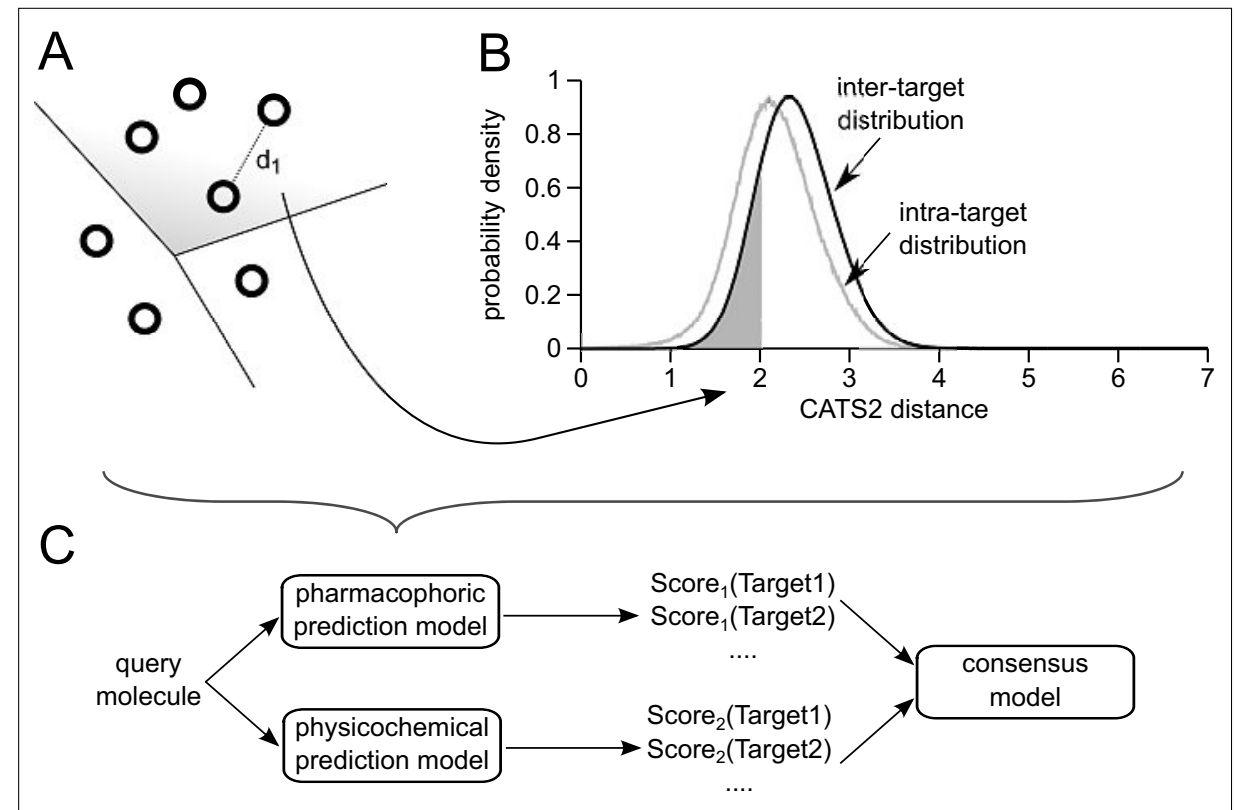

Fig. 2. (A) Self-organizing maps (SOMs) are used as a clustering approach to identify meaningful relationships between query and reference molecules (here depicted as circles). (B) We have established a protocol to transform molecular similarities into $p$ values according to background similarity distributions between molecules that are not annotated to bind the same target ("intertarget" distribution). ${ }^{[2]]}$ The indicated shaded area visualizes the $p$ value for an assumed distance of $d=2$. (C) The schematic depiction of the workflow for a SPiDER prediction. ${ }^{[11]}$ The SOM based clustering is performed twice and predictions based on pharmacophores and physicochemical properties are combined in a consensus manner. 
bination that had earlier been suggested to lead to efficient anti-tumor agents with reduced chemoresistance. ${ }^{[27]}$ The algorithm correctly and consistently identified the pharmacophoric traits in the investigated compound class. The model also served in a computational analysis to estimate the number of known drugs and bioactive compounds that might have an unknown epigenetic effect.[29] The biophysically validated top prediction for histone deacytelase (HDAC) inhibition confirmed the model's applicability. In a more compoundcentric analysis, the algorithm successfully deciphered the potential mechanism of action of potent and innovative antiplasmodial compounds. ${ }^{[30]}$ The investigated class of pyrrolopyrazines had shown potent antiplasmodial effects that were not fully explainable trough the known targets of these compounds. Thorough analysis of the predicted targets using our SOM method revealed multi-target engagement, including the inhibition of specific Plasmodium falciparum kinases.

Encouraged by these results, we investigated the concept further and employed different molecular representations of the references and query molecules. We observed that the algorithm performed similarly successful when used with physicochemical properties as a description method, however with only weak correspondence to the CATS pharmacophorebased method when looking at the performance predicting specific targets. This motivated us to combine the two methods in a consensus approach, which we named SPiDER (Fig. 2C, Table 1). [11] Different mathematical combination functions were shown to result in up to $50 \%$ increase in accuracy over individual models, which is fully in line with the improvements we observed for combinations of other cascaded machine-learning classifiers. ${ }^{[31]}$ With this tool in hand, we predicted meaningful bioactivities of both known drugs and NCEs in the absence of structural similarity to the reference ligands (Tanimoto substructure similarity <0.2). In particular, we identified G-protein coupled receptor (GPCR) engagement of compounds that structurally resemble HIV1 protease inhibitors. This biochemically confirmed prediction was unique to the SPiDER approach when compared to other, publicly available target prediction methods (Table 1).

\section{From Mathematical Models to Designer Molecules with the Desired Target Selectivity}

We have engaged in numerous early discovery programs using the ligand-based Design of Genuine Structures (DOGS) software, to generate NCEs for drug tar- gets of high therapeutic interest. ${ }^{[32]}$ DOGS implements a reaction-based algorithm comprising 83 organic reactions and a predefined library of commercially available building blocks to ensure the synthetic tractability of the computer-generated NCEs. ${ }^{[33]}$ The software grows molecules sequentially and the quality of the designed molecules, including intermediates, is evaluated by a dual ligand-based scoring scheme (ISOAK), which considers both structural and pharmacophoric features. ${ }^{[34]}$ The score computed for a newly generated virtual molecule is expressed as its similarity to a given template molecule (e.g. a known drug). The design algorithm optimizes the score by iteratively generating molecule variations, thereby attempting to mimic the features of the design template. For the similarity evaluation, different levels of abstraction from the atomistic structure may be employed, e.g. by using different molecular graph representations, which we implemented as kernel functions. ${ }^{35]}$ We employ reduced graph representations by default, acknowledging a general topological representation of the template molecules.

We initially focused on designing diverse, innovative, and potent kinase inhibitors. For example, compound 1 (Fig. 3) had been previously identified as a selective and potent $\left(\mathrm{IC}_{50}=0.2 \mathrm{nM}\right)$ type II human Polo-like kinase (hPlk-1) inhibitor (Fig. 4). ${ }^{[37]}$ Taking 1 as template we readily identified $\mathbf{2}$ as an innovative small molecule with similar biological properties to $\mathbf{1}$ $\left(\mathrm{IC}_{50} \approx 100 \mathrm{nM}\right) .^{[38]}$ Advanced exploration of the 218 de novo-designed molecules steered our efforts to uncharted chemical space, more specifically, selecting $\mathbf{3}$ as a prime candidate for biochemical profiling. Its structural similarity to the antidepressant drug fluoxetine, $\mathbf{4}$, further spurred our interest in studying the effect of both molecules against hPlk-1. ${ }^{[39]}$ In fact, compound 3 showed extraordinary ligand efficiency $(L E=0.66)$ and exquisite selectivity for hPlk-1. Fluoxetine presented comparable biochemical effects, albeit in the micromolar range. Significantly, de novo designed compound $\mathbf{3}$ also displayed kinase inhibitory activity against cancer cells at low micromolar concentrations, without affecting immortalized non-transformed cells.

An Aurora A kinase inhibitor was also productively obtained by DOGS using VX-680 (5, Fig. 3) as design template. ${ }^{[40]}$ Compound 6 was selected for biochemical profiling $\left(\mathrm{IC}_{50}=10 \mu \mathrm{M}\right)$, despite the considerable differences between the designed compound and a large set of known kinase inhibitors. In fact, similarity-based approaches for target profile prediction were not successful in identifying $\mathbf{6}$ as an Aurora A kinase inhibitor, thereby validating the use of DOGS for the purpose of scaffoldhopping. [41]

Finally, we designed vascular endothelium growth factor receptor-2 (VEGFR-2) inhibitors based on the kinase-selective template AMG-706 (7, Fig. 3). ${ }^{[42]}$ The $d e$ novo design produced a different scaffold for every second suggested molecule. We selected compound $\mathbf{8}$ for synthesis and testing because it was apparently free of intellectual-property constraints. While it inherited the overall kinase selectivity of the parent template, 8 was only a weak inhibitor of VEGFR-2 $\left(\mathrm{IC}_{50}=14 \mu \mathrm{M}\right)$. We

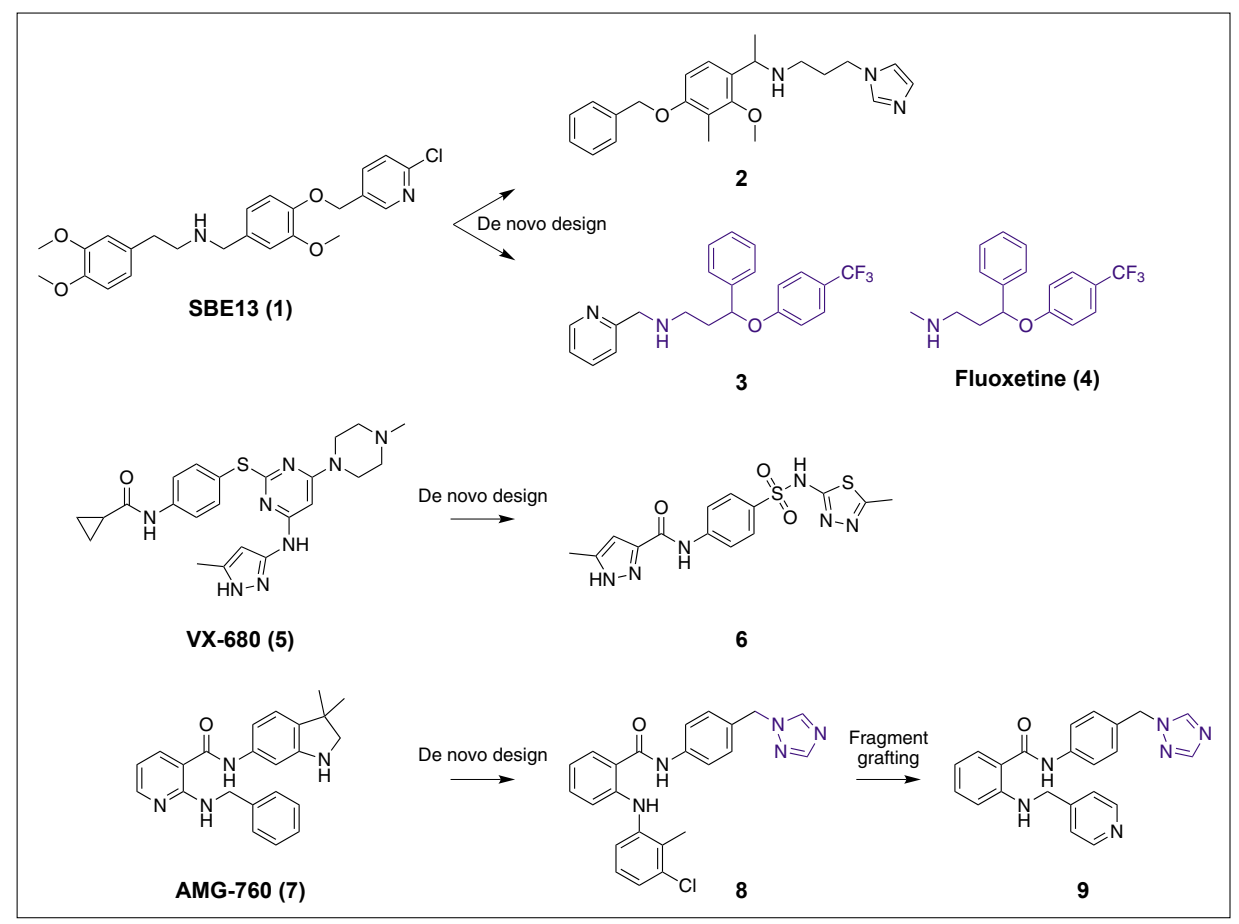

Fig. 3. Overview of de novo designed chemical entities as kinase inhibitors. 


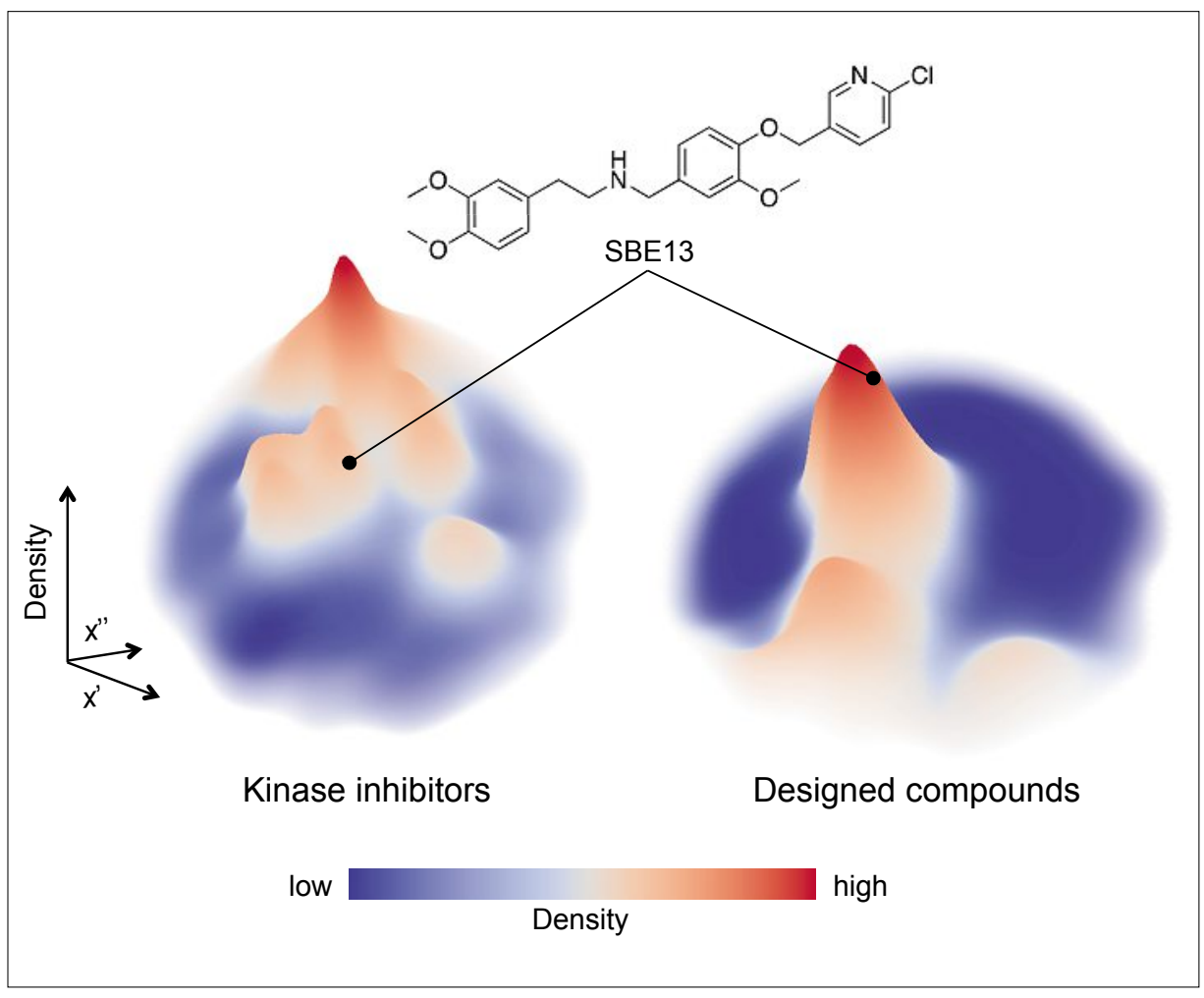

Fig. 4. Visualization of chemical space by nonlinear projection and Gaussian mixture models (LiSARD software). ${ }^{\left[{ }^{36]}\right.}$ The DOGS design template SBE13 (1), a selective type-II inhibitor of hPlk-1, is located at the border of the distribution of known kinase inhibitors. De novo generated molecules were derived from this template structure and shown to populate an extended chemical space compared to the known kinase inhibitors.

then hypothesized that a hinge binding motif was absent in $\mathbf{8}$ and that grafting a pyridyl moiety would heavily impact on its inhibitory potency. Indeed, we obtained 9 as a ligand-efficient ( $L E=0.35$ ) lownanomolar inhibitor of VEGFR-2 $\left(\mathrm{IC}_{50}=\right.$ $64 \mathrm{nM}$ ) with approximately 40-fold selectivity over VEGFR-1 $\left(\mathrm{IC}_{50}=2400 \mathrm{nM}\right)$, and astonishing overall kinase selectivity. Furthermore, the selectivity observed in biochemical assays was translated in vitro in a wound-healing assay, and negligible activity in cell lines not expressing VEGFR-2.

To design de novo GPCR ligands we developed the adaptive Molecular Ant

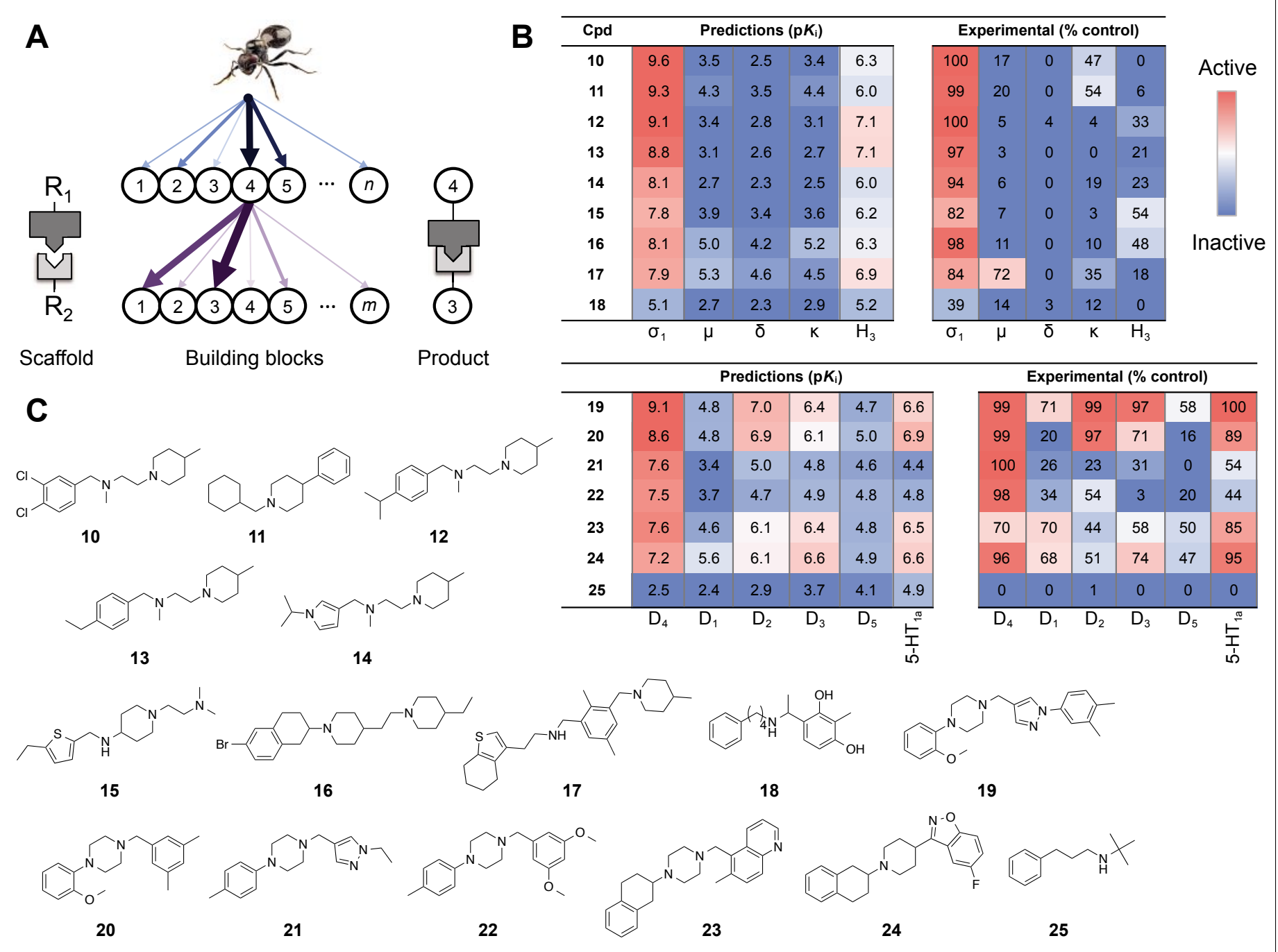

Fig. 5. De novo design of GPCR ligands. A) Schematic of the MAntA design algorithm. B) Receptor binding affinity predictions ( $\left(\mathrm{p} K_{\mathrm{i}}\right)$ and experimental data (\% control) for GPCR target panels. C) Structures of the designed ligands obtained through reductive amination. Activity data taken from ref. [43a]. 
Algorithm (MAntA), which implements a fragment prioritization approach to combinatorial chemistry, mimicking the foraging behavior of certain ant species (Fig. 5). [43,44] In a proof-of-concept study, we focused on reductive amination as a privileged reaction, and employed quantitative Gaussian Process models for scoring the generated compound libraries. ${ }^{[45]}$ With predictive bioactivity models for 640 human drug targets at hand, we prioritized compounds for synthesis and biochemical profiling fulfilling different criteria in a stepwise probation of rational polypharmacologybased de novo design. We experimentally validated the design approach by identifying innovative ligand-efficient ligands with nanomolar activities for sigma- 1 and dopamine $\mathrm{D}_{4}$ receptors, while displaying an accurately predicted multi-target binding affinity profile. Whereas for sigma- 1 the selected compounds generally presented good selectivity, for the $D_{4}$ receptor a polypharmacological profile could not be precluded for most of the selected small molecules (Fig. 5). In fact, the MAntA scoring algorithm was able to recognize subtle structural patterns and accurately predicted GPCR binding with a success rate of approximately $90 \%$. The accuracy and domain of applicability of MAntA is bound to continuously improve with time as more structure-activity relationship data becomes available. The design concept may be applied to any reaction scheme, including multistep routes and automation, providing a feasible means for rational chemical space exploration. ${ }^{[46]}$

\section{Conclusions}

Accurate and robust target prediction for NCEs has become feasible through the use of machine-learning algorithms and reliable training data. Heuristics and empirical models help in the fast prioritization of both physically available and computer-generated virtual chemical entities. Computational medicinal chemistry has greatly benefitted from the adaptation of statistical significance estimations to molecular similarity analyses, which renders de novo designed molecules acceptable for synthesis with a good chance of success. One can expect these methods to become a natural part of the medicinal chemist's toolbox in the very near future. The next phase of polypharmacological drug design will have to address the incorporation of pharmacokinetic and pharmacodynamic properties and clinical endpoints. This way, target activities might even be associated with clinical outcomes and allow for the future identification and development of personalized drugs.

\section{Acknowledgements}

ETH Zürich, the Swiss National Science Foundation (SNF grant no. 205321-134783), and the OPO Foundation Zürich funded these studies. The authors thank all members of the ETH research group for sustained support and stimulating discussions.

\section{Conflict of interest statement}

G. S. and P. S. are co-founders of inSili. com LLC, Zürich, and act as consultants in the pharmaceutical industry. G. S. is a co-founder of AlloCyte Pharmaceuticals Ltd, Basel. P. S. is the acting CEO of inSili.com LLC.

Received: June 30, 2014

[1] 'De Novo Molecular Design', Ed. G. Schneider, Wiley-VCH, Weinheim, New York, 2013.

[2] a) L. K. Buehler, PharmaGenomics 2004, 4, 24; b) C. T. Keith, A. A. Borisy, B. R. Stockwell, Nat. Rev. Drug Discov. 2005, 4, 71.

[3] a) D. G. Rudmann, Toxicol. Pathol. 2013, 41, 310; b) E. Lounkine, M. J. Keiser, S. Whitebread, D. Mikhailov, J. Hamon, J. L. Jenkins, P. Lavan, E. Weber, A. K. Doak, S. Côté, B. K. Shoichet, L. Urban, Nature 2012, $486,361$.

[4] a) X. Liu, F. Zhu, X. H. Ma, Z. Shi, S. Y. Yang, Y. Q. Wei, Y. Z. Chen, Curr. Med. Chem. 2013, 20, 1646; b) A. Koutsoukas, B. Simms, J. Kirchmair, P. J. Bond, A. V. Whitmore, S. Zimmer, M. P. Young, J. L. Jenkins, M. Glick, R. C. Glen, A. Bender, J. Proteomics 2011, 74, 2554; c) A. Bender, J. Scheiber, M. Glick, J. W. Davies, K. Azzaoui, J. Hamon, L. Urban, S. Whitebread, J. L. Jenkins, ChemMedChem 2007, 2, 861; d) P. Csermely, V. Agoston, S. Pongor, Trends Pharmacol. Sci. 2005, 26, 178; e) T. Korcsmáros, M. S. Szalay, C. Böde, I. A. Kovács, P. Csermely, Expert Opin. Drug Discov. 2007, 2, 799

[5] a) A. Spitzmüller, J. Mestres, PLoS Comput. Biol. 2013, 9, e1003257; b) K. S. Dias, C. Viegas Jr, Curr. Neuropharmacol. 2014, 12, 239; c) S. Huang, S. Kauffman, Semin. Cancer Biol. 2013, 23, 270; d) T. Hanke, F. Dehm, S. Liening, S. D. Popella, J. Maczewsky, M. Pillong, J. Kunze, C. Weinigel, D. Barz, A. Kaiser, M. Wurglics, M. Lämmerhofer, G. Schneider, L. Sautebin, M. Schubert-Zsilavecz, O. Werz, $J$. Med. Chem. 2013, 56, 9031; e) J. Lotharius, F. J. Gamo-Benito, I. Angulo-Barturen, J. Clark, M. Connelly, S. Ferrer-Bazaga, T. Parkinson, P. Viswanath, B. Bandodkar, N. Rautela, S. Bharath, S. Duffy, V. M. Avery, J. J. Möhrle, R. K. Guy, T. Wells, Malar J. 2014, 13, 143.

[6] M. L. Bolognesi, Curr. Med. Chem. 2013, 20, 1639.

[7] a) T. T. Ashburn, K. B. Thor, Nat. Rev. Drug Discov. 2004, 3, 673; b) M. J. Keiser, V. Setola, J. J. Irwin, C. Laggner, A. I. Abbas, S. J. Hufeisen, N. H. Jensen, M. B. Kuijer, R. C. Matos, T. B. Tran, R. Whaley, R. A. Glennon, J. Hert, K. L. Thomas, D. D. Edwards, B. K. Shoichet, B. L. Roth, Nature 2009, 462, 175; c) S. Dakshanamurthy, N. T. Issa, S. Assefnia, A. Seshasayee, O. J. Peters, S. Madhavan, A. Uren, M. L. Brown, S. W. Byers, J. Med. Chem. 2012, 55, 6832; d) Y. Y. Li, J. An, S. J. Jones, Genome Inform. 2006, 17, 239; e) A. Anighoro, J. Bajorath, G. Rastelli, J. Med. Chem. 2014, doi: $10.1021 / \mathrm{jm} 5006463$.

[8] G. Jin, S. T. Wong, Drug Discovery Today 2014, $19,637$.

[9] a) M. J. Keiser, J. J. Irwin, B. K. Shoichet, Biochemistry 2010, 49, 10267; b) J. Lötsch, G. Schneider, D. Reker, M. J. Parnham, P. Schneider, G. Geisslinger, A. Doehring, Trends Mol. Med. 2013, 12, 742 .
[10] M. J. Keiser, B. L. Roth, B. N. Armbruster, P. Ernsberger, J. J. Irwin, B. K. Shoichet, Nat. Technol. 2007, 25, 197.

[11] D. Reker, T. Rodrigues, P. Schneider, G. Schneider, Proc. Natl. Acad. Sci. USA 2014, 111, 4067.

[12] B. Chen, Y. Ding, D. J. Wild, PLoS Comput. Biol. 2012, 8, e1002574.

[13] A. Lagunin, A. Stepanchikova, D. Filimonov, V. Poroikov, Bioinformatics 2000, 16, 747.

[14] L. Wang, C. Ma, P. Wipf, H. Liu, W. Su, X. Q. Xie, AAPS J. 2013, 15, 395.

[15] M. Dunkel, S. Günther, J. Ahemd, B. Wittig, R. Preissner, Nucleic Acids Res. 2008, 36, W55.

[16] H. Li, Z. Gao, L. Kang, H. Zhang, K. Yang, K. Yu, X. Luo, W. Zhu, K. Chen, J. Shen, X. Wang, H. Jiang, Nucleic Acids Res. 2006, 34, W219.

[17] a) D. Rognan, Drug Discovery Today Technol. 2013, 10, e403; b) J. Meslamani, R. Bhajun, F. Martz, D. Rognan, J. Chem. Inf. Model. 2013, 53, 2322; c) S. Renner, C. Schwab, J. Gasteiger, G. Schneider, J. Chem. Inf. Model. 2006, 46, 2324.

[18] a) D. E. Patterson, R. D. Cramer, A. M. Ferguson, R. D. Clark, L. E. Weinberger, $J$. Med. Chem. 1996, 39, 3049; b) P. Willett, Mol. Inf. 2014, 33, 403.

[19] M. Vogt, J. Bajorath, Methods Mol. Biol. 2011, $672,159$.

[20] P. Schneider, Y. Tanrikulu, G. Schneider, Curr. Med. Chem. 2009, 16, 258.

[21] a) T. Kohonen, Biol. Cybern. 1982, 43, 59; b) T. Kohonen, 'Self-Organizing Maps', Springer, Berlin, Heidelberg, 3rd ed. 2001.

[22] a) J. Gasteiger, X. Li, Angew. Chem. Int. Ed. Engl. 1994, 33, 643; b) J. Zupan, J. Gasteiger, 'Neural Networks in Chemistry and Drug Design', Wiley-VCH, Weinheim, 2nd ed. 1999.

[23] G. Schneider, W. Neidhart, T. Giller, G. Schmid, Angew. Chem. Int. Ed. 1999, 38, 2894.

[24] P. Schneider, K. Stutz, L. Kasper, S. Haller, M. Reutlinger, F. Reisen, T. Geppert, G. Schneider, Pharmaceuticals 2011, 4, 1236.

[25] G. Schneider, Y. Tanrikulu, P. Schneider, Future Med. Chem. 2009, 1, 213.

[26] The SOM was generated using the software molmap and the COBRA v14.5 collection of reference compounds (inSili.com LLC, Zürich). We trained a toroidal map containing $20 \times 10$ clusters ('neurons') with an initial Gaussian neighborhood adaptivity radius $=8$ and linear decay of momentum over 105 cycles. The network graph was visualized with CytoScape v3.1.1 (http://www.cytoscape.org/).

[27] M. Reutlinger, C. P. Koch, D. Reker, N. Todoroff, P. Schneider, T. Rodrigues, G. Schneider, Mol. Inf. 2013, 32, 133.

[28] a) S. Renner, M. Hechenberger, T. Noeske, A. Böcker, C. Jatzke, M. Schmuker, C. G. Parsons, T. Weil, G. Schneider, Angew. Chem. Int. Ed. 2007, 46, 5336; b) T. Noeske, D. Trifanova, V. Kauss, S. Renner, C. G. Parsons, G. Schneider, T. Weil, Bioorg. Med. Chem. 2009, 17, 5708; c) T. Noeske, B. C. Sasse, H. Stark, C. G. Parsons, T. Weil, G. Schneider, ChemMedChem 2006, 1, 1066; d) G. Schneider, M. Nettekoven, J. Comb. Chem. 2003, 5, 233.

[29] J. Lötsch, G. Schneider, D. Reker, M.J. Parnham, P. Schneider, G. Geisslinger, A. Doehring, Trends Mol. Med. 2013, 12, 742.

[30] D. Reker, M. Seet, M. Pillong, C. P. Koch, P. Schneider, M.C. Witschel, M. Rottmann, C. Freymond, R. Brun, B. Schweizer, B. Illarionov, A. Bacher, M. Fischer, F. Diederich, G. Schneider, Angew. Chem. Int. Ed. 2014, 53, 7079.

[31] a) C. P. Koch, A. M. Perna, S. Weissmüller, S. Bauer, M. Pillong, R. B. Baleeiro, M. Reutlinger, G. Folkers, P. Walden, P. Wrede, J. A. Hiss, Z. Waibler, G. Schneider, ACS Chem. Biol. 2013, 8,1876 ; b) C. P. Koch, A. M. Perna, M. Pillong, N. K. Todoroff, P. Wrede, G. Folkers, J. A. Hiss, 
G. Schneider, PLoS Comput. Biol. 2013, 9, e1003088; c) A. Givehchi, G. Schneider, Mol. Divers. 2005, 9, 371 .

[32] a) T. Rodrigues, G. Schneider, Synlett 2014, 25 , 170; b) G. Schneider, J. Comput. Aided Mol. Des. 2012, 26, 115; c) G. Schneider, Chimia 2012, 66, 120; d) G. Schneider, Mol. Inf. 2014, $33,397$.

[33] M. Hartenfeller, H. Zettl, M. Walter, M. Rupp, F. Reisen, E. Proschak, S. Weggen, H. Stark, G. Schneider, PLoS Comput. Biol. 2012, 8, e1002380.

[34] M. Rupp, E. Proschak, G. Schneider, J. Chem. Inf. Model. 2007, 47, 2280 .

[35] M. Rupp, G. Schneider, Mol. Inf. 2010, 29, 266.

[36] a) M. Reutlinger, W. Guba, R. E. Martin, A. I. Alanine, T. Hoffmann, A. Klenner, J. A. Hiss, P. Schneider, G. Schneider, Angew. Chem. Int. Ed. 2011, 50, 11633; b) M. Reutlinger, G Schneider, J. Mol. Graph. Model. 2012, 34, 108.
[37] S. Keppner, E. Proschak, G. Schneider, B. Spänkuch, ChemMedChem 2009, 4, 1806.

[38] G. Schneider, T. Geppert, M. Hartenfeller, F. Reisen, A. Klenner, M. Reutlinger, V. Hähnke, J. A. Hiss, H. Zettl, S. Keppner, B. Spänkuch, P. Schneider, Future Med. Chem. 2011, 3, 415.

[39] B. Spänkuch, S. Keppner, L. Lange, T. Rodrigues, H. Zettl, C. P. Koch, M. Reutlinger, M. Hartenfeller, P. Schneider, G. Schneider, Angew. Chem. Int. Ed. 2013, 52, 4676.

[40] T. Rodrigues, F. Roudnicky, C. P. Koch, T. Kudoh, D. Reker, M. Detmar, G. Schneider, Chem. Sci. 2013, 4, 1229.

[41] G. Schneider, Drug Discovery Today Technol. 2013, 10, e453.

[42] T. Rodrigues, T. Kudoh, F. Roudnicky, Y. F Lim, Y. C. Lin, C. P. Koch, M. Seno, M. Detmar, G. Schneider, Angew. Chem. Int. Ed. 2013, 52, 10006.

[43] a) M. Reutlinger, T. Rodrigues, P. Schneider, G. Schneider, Angew. Chem. Int. Ed. 2014,
53, 4244; b) J. A. Hiss, M. Hartenfeller, G. Schneider, Curr. Pharm. Des. 2010, 16, 1656.

[44] G. Schneider, M. Hartenfeller, M. Reutlinger, Y. Tanrikulu, E. Proschak, P. Schneider, Trends Biotechnol. 2009, 27, 18.

[45] a) M. Reutlinger, T. Rodrigues, P. Schneider, G. Schneider, Angew. Chem. Int. Ed. 2014, 53 , 582; b) J. A. Hiss, M. Reutlinger, C. P. Koch, A. M. Perna, P. Schneider, T. Rodrigues, S. Haller, G. Folkers, L. Weber, R. B. Baleeiro, P. Walden, P. Wrede, G. Schneider, Future Med. Chem. 2014, 6, 267.

[46] T. Rodrigues, P. Schneider, G. Schneider, Angew. Chem. Int. Ed. 2014, 53, 5750. 